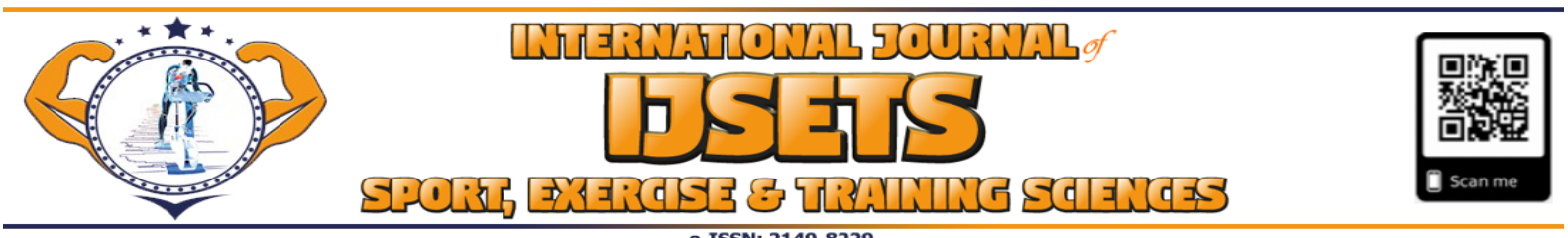

\title{
Kinetics of cytokines IL-6, IL-10 and TNF- $\alpha$ and their relationship with serum IGF-I and IGFBP-3 concentrations in adolescent swimmers throughout a training season
}

\author{
Marcela PIRES ${ }^{1}$, Hugo Tourinho FILHO ${ }^{2}$, Eike KOHAMA ${ }^{3}$, Rafael FORNEL ${ }^{2}$, Rodrigo \\ CUSTÓDIO $^{1}$, Carlos Eduardo Martinelli JUNIOR ${ }^{1}$
}

\begin{abstract}
Aim: The objective of this study was to analyze the kinetics of the IL-6, IL-10, and TNF- $\alpha$ cytokines and their relationship with the serum IGF-I and IGFBP-3 levels of adolescent swimmers throughout a training season.

Methods: Nine male adolescent swimmers aged 16 to 19 years old, classified in stages 4-5 for pubic hair growth on the Tanner scale, were recruited as the study sample. All the procedures took place in the early phase (extensive phase), intermediate phase (intensive phase), and final phase (tapering) of the athletes' season. At the beginning of each stage, the athletes were evaluated as follows: an anthropometric evaluation and blood samples were taken before and after a standardized training session (STS).

Results: It was possible to verify a strong and inversely proportional relationship between serum levels of IL- 6 and IGF-I during the most intensive phase of training and an increase in serum concentrations of IGF-I and IL-10 during the tapering phase. Regarding the acute effects of training it was possible to identify differentiated kinetics only for the IGF-I values during the intensive phase and for the IL-10 values during the tapering phase.

Conclusion: IGF-I, IGFBP-3, and IL-10 concentrations proved sensitive to both the acute or chronic effects of exercise. Thus, it can be suggested that these components may be used as important markers of the training condition of adolescent swimmers during their preparation throughout a season.
\end{abstract}

Keywords
biomarkers,
cytokines,
GH/IGF-I axis,
swimming,
adolescents

$\underline{\text { Article Info }}$

Received: 15.07 .2020

Accepted: 30.11 .2020

Online Published: 15.12 .2020

DOI: $10.18826 /$ useeabd. 843434

\section{INTRODUCTION}

Intense exercise affects the production of cytokines, a group of intercellular signaling glycoproteins associated with the control, amplification, coordination, and regulation of the magnitude and duration of immune responses, also known to directly inhibit the anabolic activity of the Growth Hormone/Insulin-like Growth Factor axis (GH/IGF-I axis). These molecules are secreted primarily by macrophages and lymphocytes in response to injury or infection but can also be released by active musculature, adipose tissue, and endothelial cells (Martinelli Jr, Custódio \& Oliveira, 2008; Scheett, Mills, Ziegler, Stoppani \&Cooper,1999; Moldoveanu, Sheph \& Shek, 2001).

High intensity training seems to cause an important metabolic modulation, characterized by an elevation of inflammatory markers (cytokines) and, consequently, suppression of the GH/IGF-I axis (Silva, Goldberg, Teixeira \& Marques, 2004). The cytokines Interleukin-6 (IL-6), Interleukin-1 $\beta$ (IL$1 \beta$ ), Interleukin-1 ra (IL-1ra), and tumor necrosis factor- $\alpha$ (TNF- $\alpha$ ) are best known for directly inhibiting the anabolic activity of the GH/IGF-I axis (Nemet, Oh, Kim, Hill \& Cooper, 2002; Nemet, RoseGottron, Mills \& Cooper, 2003). On the other hand, IL-10 that acts in the anti-inflammatory process, is not related to the suppression of the GH/IGF-I axis after intense training sessions (Eliakim, Cooper \& Nemet, 2014).

In a pioneering study by Scheett et al. (Sheett et al., 1999), a significant increase in IL-6, TNF- $\alpha$, and IL-1ra cytokines was observed, accompanied by a reduction in circulating IGF-I levels and an increase in IGFBP-1, a binding protein known to inhibit the anabolic action of IGF-I, after a series of intense exercises performed by prepubescent children.

\footnotetext{
The role and contributions of each authors as in the section of IJSETS Writing Rules "Criteria for Authorship" is reported that: All the authors have accepted responsibility for the entire content of this submitted manuscript and approved submission.

${ }^{1}$ Department of Pediatrics, Ribeirao Preto Medical School, University of São Paulo, Ribeirao Preto, Brazil.

${ }^{2}$ Corresponding author: School of Physical Education and Sport of Ribeirao Preto, University of São Paulo, Ribeirao Preto, Brazil. tourinho@usp.br ORCID ID: 0000-0001-6517-064X

3Post-graduate Program in Rehabilitation and Functional Performance, Ribeirao Preto Medical School, University of São Paulo, Ribeirao Preto, Brazil.
} 
In the same line of investigation, when evaluating the effects of intense exercise on the kinetics of cytokines and growth mediators in adolescents practicing Greco-Roman wrestling, Nemet et al. (2002) found a decrease in circulating anabolic factors total IGF-I and bound IGF-I and a significant elevation in cytokines IL-6, TNF- $\alpha$, IL-1 $\beta$, and IL-1ra, in addition to a significant increase in IGFBP-1 levels. According to the authors, this framework resembles the catabolic state caused by septicemia and burns.

Several studies have examined the reduction in IGF-I or the GH/IGF-I axis after a series of intense exercises, however there is still a lack of data on the behavior of anabolic and catabolic mediators over a training season (Nindl et al., 2001; Nemet \& Eliakim, 2010; Eliakim \& Nemet, 2010; Eliakim, Cooper $\&$ Nemet, 2014; Tourinho Filho et al., 2017).

Following this line of reasoning, Eliakim et al. (2014) suggest that more field studies are needed to better understand the effects of training sessions, which are part of the daily programs of prepubertal children and adolescents, and their real role in the adaptations brought about by this training over a season.

In a study performed using adolescent swimmers over a season, it was possible to observe a biphasic behavior of IGF-I, with a significant drop in serum concentrations in the intensive training phase and an increase in these concentrations from the intensive phase to the tapering phase. However, IGFBP-3 showed more discrete variations and increased significantly from the intensive phase to the tapering phase, although it was not clear in the study what had caused this kinetics (Tourinho Filho et al., 2017).

Furthermore, what causes a decrease in IGF-I levels during periods of intense training or what should be an adequate elevation of this hormone during the tapering and reduced training phases remain controversial (Eliakim \& Nemet, 2013).

Thus, the objective of this study was to analyze the kinetics of the IL-6, IL-10, and TNF- $\alpha$ cytokines and their relationship with the serum IGF-I and IGFBP-3 levels of adolescent swimmers throughout a training season.

\section{METHOD}

\section{Participants}

A total of nine male adolescent swimmers aged 16 to 19 years old (18.22 \pm 1.09$)$, classified in stages 45 for pubic hair growth on the Tanner scale (Tanner, 1962), who were regularly exposed to training sessions over a season and with a technical level corresponding to national elite, were recruited as the study population.

The sample size was determined by convenience, according to the availability and acceptance of the athletes to participate in the study.

Adolescent swimmers were informed verbally and in writing, through the Informed Consent Form (signed by the parents or guardians and signed by the adolescents), regarding the procedures that would be adopted in the research. The study protocol conforms to the ethical guidelines of the 1975 Declaration of Helsinki and it was approved by the local Ethics Committee.

\section{Data collection}

All the procedures in this research took place in the early phase (extensive phase), intermediate phase (intensive phase), and final phase (tapering) of the athletes' season. At the beginning of each stage, the athletes were evaluated as follows: an anthropometric evaluation was carried out to determine body composition (lean mass and percentage of fat) and blood samples were taken before and after a standardized training session (STS).

Blood collection was performed before the start of the STS, preceded by 30 minutes of rest, and 60 minutes after the end of the session. All collections took place in the afternoon between $3 \mathrm{pm}$ and $5 \mathrm{pm}$. Subsequently, the samples were centrifuged for serum separation and stored at $-80^{\circ} \mathrm{C}$.

\section{Immunoassays}

Determination of serum $I L-6, I L-10$, and $T N F-\alpha$ :The serum concentrations of IL-6, IL-10, and TNF$\alpha$ cytokines (intensive phase and tapering) were determined using Luminex XMAPTM (MAP $=$ Multiple Analyte Profiling) technology, according to the manufacturer's specifications using Cat kit HCYTOMAG60K-03 (IL6, IL10, and TNF- $\alpha$ ) - Lot: 2690187 - Exp. 31/10/2016 - Supplier Millipore. 
The reading was performed using Luminex 200 xPonent and Analyst software version 4.2 and serum concentrations were given in $\mathrm{pg} / \mathrm{mL}$.

All the dosages described above were performed in the same assay at the Laboratory Specialized in Scientific Analyses.

Serum IGF-I and IGFBP-3 concentrations: Serum concentrations of IGF-I and IGFBP-3 (Immulite 2000, Siemens, Los Angeles, CA, USA) were determined by specific immunoassays using commercial kits. All samples were analyzed in duplicate within the same assay. The intra-assay variations were 2.4 $\%$ for IGF-I and $2.3 \%$ for IGFBP-3. Assay sensitivity was $5 \mathrm{ng} / \mathrm{ml}$ for IGF-I and $0.1 \mathrm{mg} / \mathrm{l}$ for IGFBP-3. Anthropometric measures and body composition

Body mass was determined using an electronic scale (Lucastec - Ple 180) and triceps, subscapular, suprailiac, and abdominal skinfold measurements were obtained using an adipometer skinfold caliper (Cescorf), according to the standards set by Behnke and Wilmore (Behnke \& Wilmore, 1974). Body composition was determined according to the equation proposed by Boileau, Lohman and Slaughter (Boileau, Lohman \& Slaughter, 1985).

\section{Periodization of training throughout the season}

In order to quantify in meters, the training loads applied in the different phases of the training season, the following divisions were used: Zone 1 (Z1) - subliminal intensity, Zone 2 (Z2) - intensity relative to anaerobic threshold, and Zone $3(\mathrm{Z3})$ - supraliminal intensity or $\mathrm{VO}_{2 \text { peak }}$ intensity.

At the end of the extensive phase it was possible to note a greater quantity of training in Z1. By the end of the intensive phase, $Z 1$ training was reduced and a greater amount of $Z 3$ was applied until the start of the tapering phase. During the tapering phase, the number of meters swum in both Z1 and Z3 were reduced in each training session. A reduction in the number of meters swum in Z2 was observed at the start of the intensive phase, stabilizing from then until the end of the tapering phase (Figure 1).

Quantification of the training loads demonstrated that the most intensive training occurred during the intensive phase, while the largest volume of training took place during the extensive phase. There was a reduction in both the volume (Z1) and intensity (Z3) of the training loads during the tapering phase.

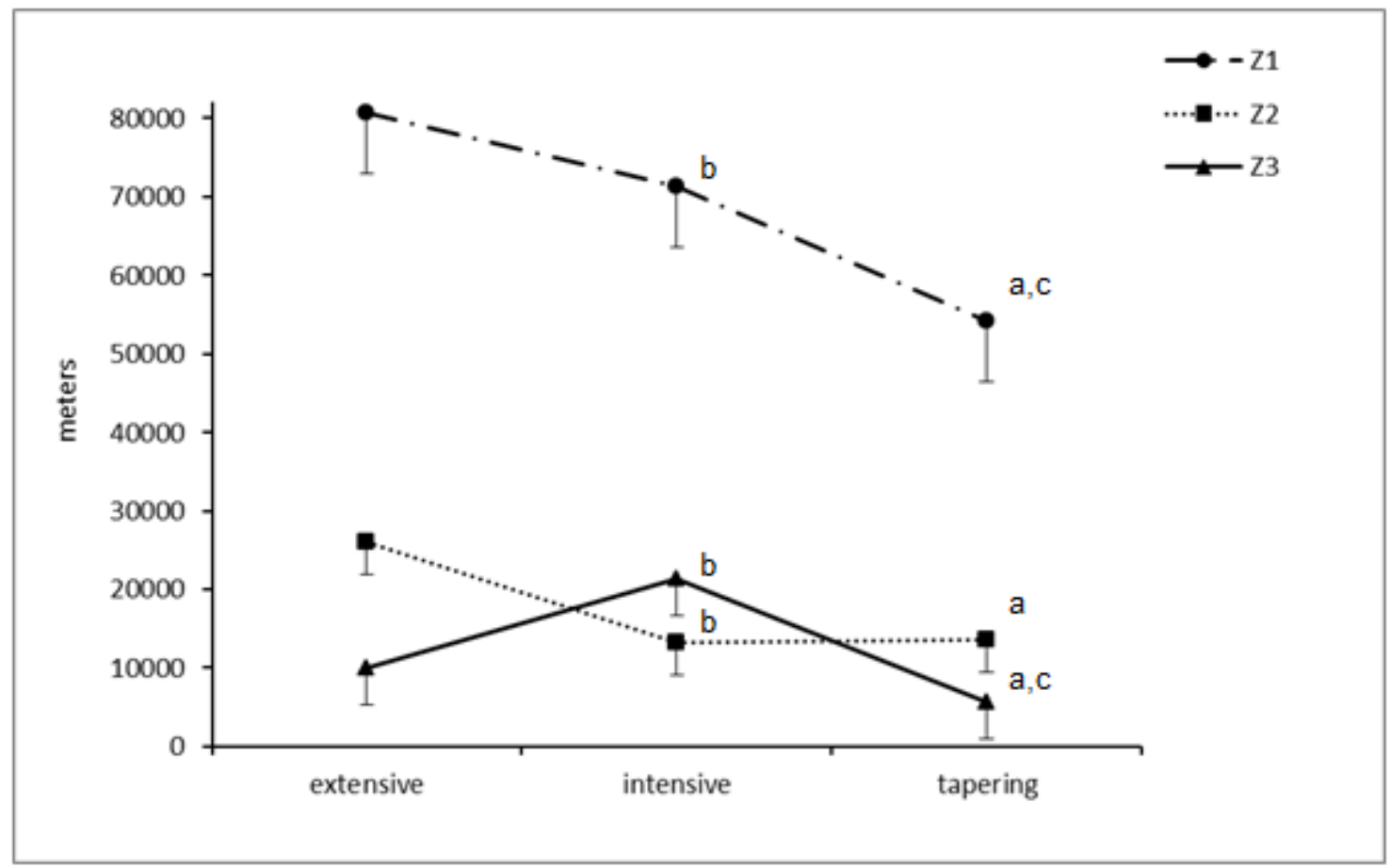

Fig. 1. Training load (meters) distribution in the different phases of a training season: subliminal (Z1), relative to anaerobic threshold (Z2), and supraliminal (Z3) intensity. The most intensive training (Z3) occurred during the intensive phase, while the largest volume of training (Z1) took place during the extensive phase. There was a reduction in both the volume (Z1) and intensity $(\mathrm{Z} 3)$ of the training loads during the tapering phase. A reduction in the meterage swum in Z2 was observed at the start of the intensive phase, stabilizing from then until the end of the tapering phase. Significant differences are labelled as: (a) extensive vs. tapering; (b) extensive vs. intensive; and (c) intensive vs. tapering. 
During the periodization of the 19 weeks of training, seven weeks were dedicated to the extensive phase, seven to the intensive phase, and five to the tapering phase.

The relationship between volume and intensity of training used throughout the season followed the recommendations of Maglischo (2010), Seiler \& Kjerland (2006), and Esteve-Lanao, Juan, Earnest, Foster \& Lucia (2005), however, training was determined exclusively by the coach, with no interference from the researchers.

\section{Standardized training session (STS)}

The standardized training session (STS) used for data collection in the different phases of training consisted of a $1500 \mathrm{~m}$ warm up performed exclusively in the front crawl stroke at moderate intensity (subjectively determined by the swimmers) followed by the main part of the session, which consisted of 10 maximum effort sprints of $50 \mathrm{~m}$ in the front crawl stroke, with a two-minute interval between sprints. This style of training was chosen for its high intensity.

At the end of the training session, the athletes were instructed to swim $1000 \mathrm{~m}$ at moderate intensity (subjectively determined by the swimmers), alternating between swimming drills and exercises for arms and legs.

\section{4h Diet Recall}

Multiple surveys were administered aiming to quantify the amount of food consumed in the 24-hour period prior to the interview. To calculate the data obtained from the multiple 24-hour dietary recalls, the DietWin ${ }^{\circledR}$ program was used, which is a support system for nutritional assessment and diet prescription. The parameters established by the American Dietetic Association and Dietary Reference Intakes (DRIs) for age and level of physical activity performed were used to analyze the data obtained (Marchioni, Slater \& Fisberg, 2004).

\section{Statistical treatment}

The Shapiro-Wilk test was used to test the normality of the data. To analyze the kinetics of the cytokines IL-6, IL-10, and TNF- $\alpha$ in the different phases of the training (extensive vs. intensive vs. tapering) and before and after the standardized training session (pre vs. post), Friedman and Wilcoxon non-parametric tests were used, respectively, adopting a significance level of 0.05 . As the Friedman test does not present a Post-Hoc test, if statistical differences were identified in the serum concentrations of IL-6, IL-10, and TNF- $\alpha$ throughout the season, the Wilcoxon test was applied in order to identify where such differences occurred.

For the statistical analysis of cytokines after training sessions in the tapering phase (third moment), eight athletes were considered due to the insufficient sample size of one athlete. In addition, specifically regarding IL-10, one swimmer was also excluded from the analyses (due to gastroenteritis), leaving a total of seven athletes in the tapering phase.

In addition, the correlation between two variables was analyzed using Spearman's correlation coefficient. All analyses were performed using STATA version 12.0.

\section{RESULTS}

The main effects of the swimming training season on serum concentrations of IL-6, IL-10, TNF- $\alpha$, IGFI, and IGFBP-3, and on body composition and correlation values observed in the different phases of the training of the adolescent swimmers, are summarized in Tables 1, 2 and 3 and in Figures 2, 3, 4, and 5.

Table 1 - Mean and standard deviation values for body mass, lean mass, and fat percentage of the swimmers in the different phases of training throughout the season.

\begin{tabular}{ccccc} 
& & & \\
VARIABLES & Extensive & PHASES & Tapering & Intensive \\
\hline Body Mass (kg) & $73.53 \pm 6.53$ & $74.74 \pm 7.93$ & $72.69 \pm 6.94$ & 0.84 \\
Lean Mass (kg) & $65.05 \pm 5.24$ & $65.92 \pm 5.61$ & $64.78 \pm 5.03$ & 0.90 \\
Fat Percentage & $11.40 \pm 3.80$ & $11.61 \pm 3.02$ & $10.71 \pm 2.79$ & 0,99 \\
\hline
\end{tabular}

$\mathrm{Kg}=$ Kilograms

There were no statistically significant differences in the values of body mass, lean mass, or percentage of fat measured in the swimmers throughout the season.

In relation to IL-6, IL-10, and TNF- $\alpha$ cytokines analyzed intra-phase (pre vs. post), significant differences were found in the IL-10 concentrations in the results obtained in the tapering phase $(1.25 \pm$ 
0.48 vs. $5.09 \pm 4.66, p=0.0156$ ). For the IL- 6 and TNF- $\alpha$ values, no statistical differences were observed when comparing the values before and after the standardized training session.

In the analysis performed during the season (inter-phases) it was possible to observe significant differences between the IL-10 values. Differences were found specifically among the post-training intensive phase IL-10 values when compared to the post-tapering IL-10 values $(2.62 \pm 1.68$ vs. $5.09 \pm$ 4.66, $\mathrm{p}=0.0391)$. A significant difference in the $\Delta_{\mathrm{IL}-10}$ can also be observed in the different phases of the training $\left(\Delta_{\text {IL-10INTENSIVE vS }} \Delta_{\text {IL-10TAPERING }}\right.$ ), specifically between the $\Delta_{\text {IL-10 }}$ values of the intensive phase and the $\Delta_{\mathrm{IL}-10}$ values of the tapering phase $(1.05 \pm 2.09$ vs. $4.18 \pm 4.66$; $\mathrm{p}=0.0469)$ (Table 2; Figura 2). No significant differences were found in any of the other comparisons performed during the different phases of the season (inter-phases).

Table 2. Effects of the standardized training session on the serum concentrations of IL-10, IL-6, and TNF- $\alpha$ cytokines observed in the swimmers at different stages of training throughout the season.

\begin{tabular}{|c|c|c|c|}
\hline \multirow{2}{*}{ VARIABLES } & \multicolumn{2}{|c|}{ PHASES } & \multirow[b]{2}{*}{ Tapering } \\
\hline & Extensive & Intensive & \\
\hline \multicolumn{4}{|l|}{ IL-10 (pg/mL) } \\
\hline Pre-training & -------- & $1.57 \pm 1.04$ & $1.25 \pm 0.48^{+}$ \\
\hline Post-training* & -------- & $2.62 \pm 1.68$ & $5.09 \pm 4.66$ \\
\hline$\Delta_{\mathrm{IL-10}} *$ & -------- & $1.05 \pm 2.09$ & $4.18 \pm 4.66$ \\
\hline \multicolumn{4}{|l|}{ IL-6 (pg/mL) } \\
\hline Pre-training & -------- & $1.13 \pm 1.44$ & $0.96 \pm 0.90$ \\
\hline Post-training & -------- & $1.50 \pm 0.81$ & $1.61 \pm 0.89$ \\
\hline$\Delta_{\text {IL-6 }}$ & -------- & $0.37 \pm 1.63$ & $0.65 \pm 1.10$ \\
\hline \multicolumn{4}{|l|}{ TNF- $\alpha(\mathrm{pg} / \mathrm{mL})$} \\
\hline Pre-training & -------- & $5.88 \pm 2.79$ & $5.50 \pm 5.05$ \\
\hline Post-training & -------- & $5.43 \pm 4.04$ & $5.50 \pm 4.67$ \\
\hline$\Delta_{\text {TNF- } \alpha}$ & -------- & $-0.45 \pm 2.77$ & $0.025 \pm 6.42$ \\
\hline
\end{tabular}

${ }^{*} \mathrm{p} \leq 0.05$ for inter-phase comparisons (extensive vs. intensive vs. tapering)

${ }^{+} \mathrm{p} \leq 0.05$ for intra-phase comparisons (pre vs. post).

$\Delta=$ (post-training session dosage-pre-training session dosage).

$\mathrm{pg} / \mathrm{ml}=$ picogram $/$ milliliter.

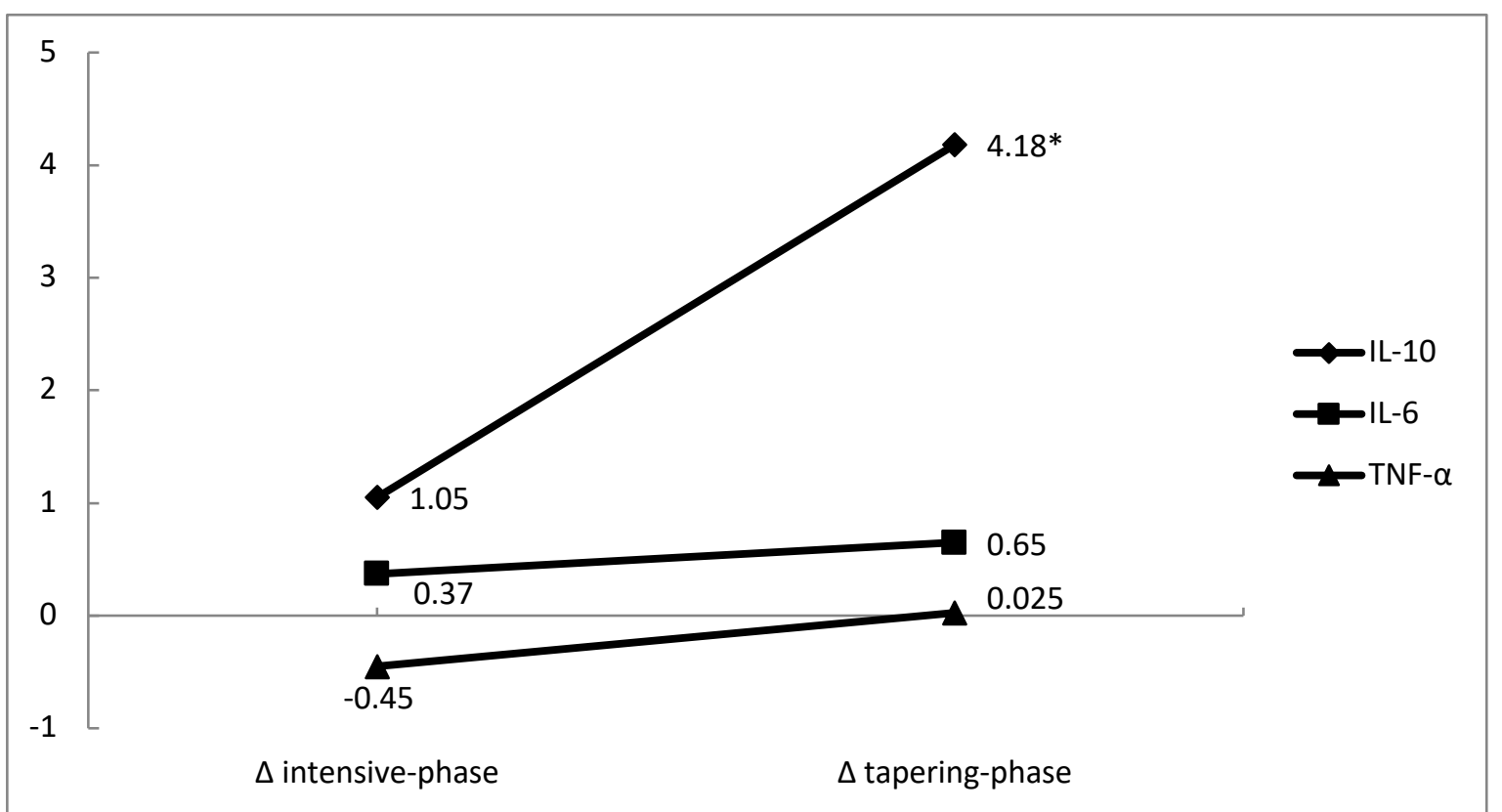

Fig. 2. Comparison between delta values of the serum IL-10, IL-6, and TNF- $\alpha$ concentrations in the intensive and tapering phases of training of the adolescent swimmers throughout the season. " $\mathrm{p} \leq 0.05$ for inter-phase comparisons (intensive vs. tapering).

Figure 3 enables the practically stable kinetics of TNF- $\alpha$ concentrations in the different phases of the season to be observed, as well as a significant increase in intra-phase (pre vs. post) IL-10 concentrations in the tapering phase, and a discrete variation, without statistical significance, in intraphase concentrations of IL- 6 both in the specific phase and in the tapering phase. 


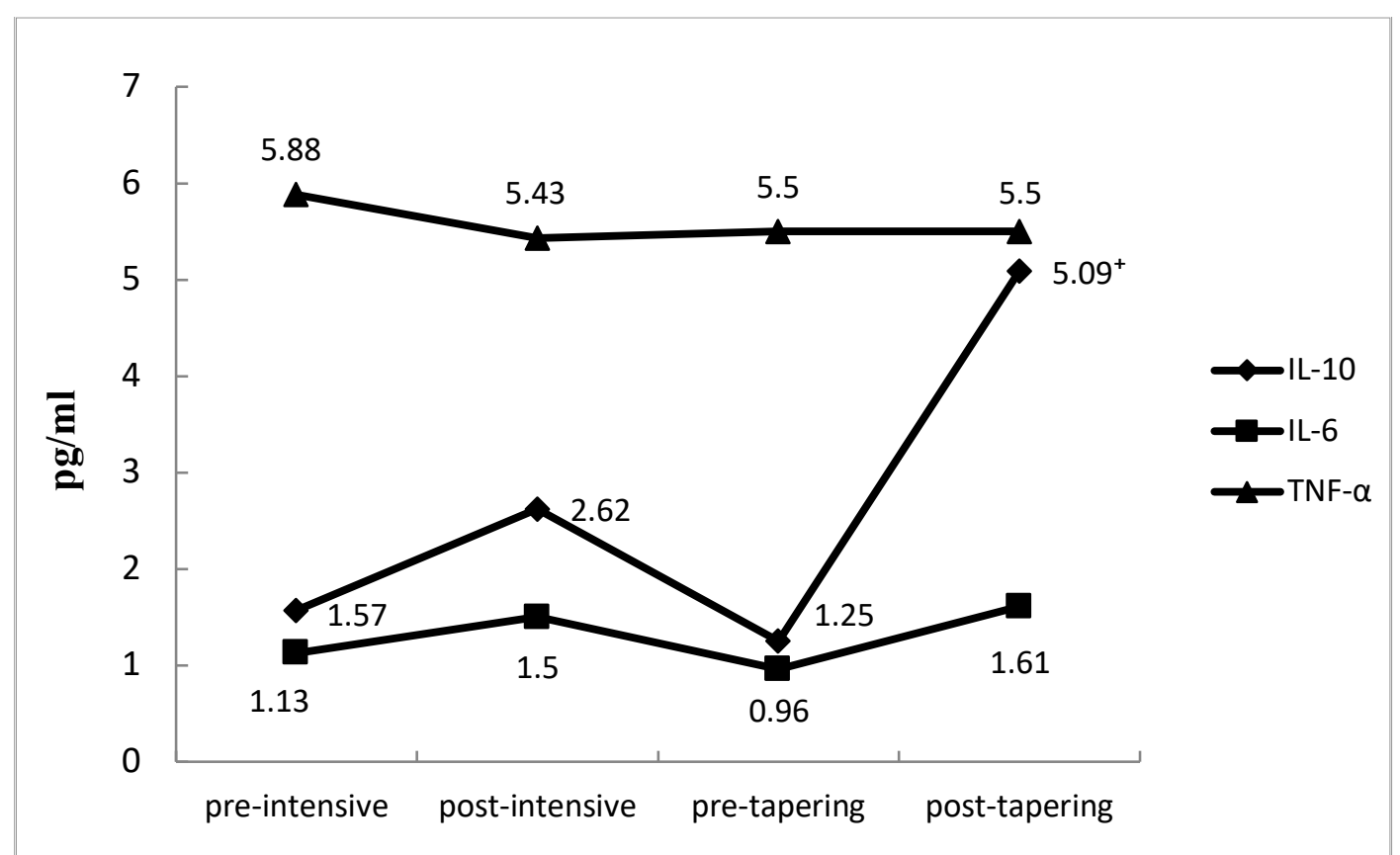

Fig. 3. Kinetics of serum concentrations of IL-10, IL-6, and TNF- $\alpha$ at different stages of training and before (pre) and after (post) the standardized training session.

Intra-phase (pre vs. post) IGF-I concentrations during the intensive phase were significantly lower post-training ( $342 \pm 58$ vs $\left.298 \pm 36 ; \Delta_{\text {IGF-I }}=44 ; \mathrm{p}=0.018\right)$. No significant difference was observed in intraphase IGF-I concentrations during the extensive or tapering phases.

Throughout the season analysis (inter-phases) revealed significant differences ( $320 \pm 40$ vs $298 \pm 36$ vs $359 \pm 94 ; p=0.04$ ) in post-training IGF-I concentrations between the different phases of training (postextensive IGF-I vs. post-intensive IGF-I vs. post-tapering IGF-I), in which post-training IGF-I concentrations in the intensive phase (post-intensiveIGF-I) were significantly lower $(p=0.004)$ than at the end of the tapering phase (post-tapering IGF-I). No significant difference was observed in pretraining IGF-I concentrations between the different phases of the season.

IGFBP-3 concentrations were significantly different $(\mathrm{p} \leq 0.05)$ between phases. Pre and posttraining concentrations varied significantly $(4.82 \pm 0.73$ vs $4.81 \pm 0.61$ vs $4.93 \pm 0.74 ; p=0.048$ and $4.74 \pm 0.71$ vs $4.67 \pm 0.47$ vs $5.00 \pm 0.71 ; p=0.035$, respectively) between the extensive, intensive, and tapering phases.

The Wilcoxon test revealed that there were significant differences $(4.74 \pm 0.71$ vs $5.00 \pm 0.71$; $\mathrm{p}=0.015$ ) between post-training IGFBP-3 levels in the extensive (post-extensive IGFBP-3) and tapering phases (post-tapering IGFBP-3). The Wilcoxon test was unable to identify where the differences in pretraining concentrations (pre IGFBP-3) occurred between the different phases (Table 3).

Table 3. Mean \pm SD serum IGF-I and IGFBP-3 concentrations in the swimmers before and after (pre vs. post) a standardized training session in the different phases of a training season.

\begin{tabular}{|c|c|c|c|}
\hline \multicolumn{4}{|c|}{ PHASES } \\
\hline & Extensive & Intensive & Tapering \\
\hline \multicolumn{4}{|l|}{ IGF-I (ng/mL) } \\
\hline Pre-training & $329 \pm 44$ & $342 \pm 58^{+}$ & $349 \pm 65$ \\
\hline Post-training * & $320 \pm 40$ & $298 \pm 36$ & $359 \pm 94$ \\
\hline$\Delta_{\text {IGF-I }}$ & $-9.11 \pm 14.90$ & $-43.33 \pm 47.32^{+}$ & $9.22 \pm 51.20$ \\
\hline \multicolumn{4}{|l|}{ IGFBP-3 (mg/l) } \\
\hline Pre-training & $4.82 \pm 0.73$ & $4.81 \pm 0.61$ & $4.93 \pm 0.74$ \\
\hline Post-training * & $4.74 \pm 0.71$ & $4.67 \pm 0.47$ & $5.00 \pm 0.71$ \\
\hline$\Delta_{\text {IGFBP-3 }}$ & $-0.08 \pm 0.77$ & $-0.14 \pm 0.64$ & $0.07 \pm 0.37$ \\
\hline
\end{tabular}

${ }^{*} \mathrm{P} \leq 0.05$ for comparison between phases (extensive vs. intensive vs. tapering).

${ }^{+} \mathrm{P} \leq 0.05$ for intra-phase comparison (pre vs. post).

$\Delta=$ (post-training concentrations - pre-training concentrations)

SD: standard deviation 
Regarding the IL-6 and IGF-I values, there is a strong and inversely proportional correlation between the absolute values of the serum concentrations of post-intensive IGF-I and the absolute values of the serum concentrations of post-intensive IL-6 $(r=-0.76, p=0.04)$, indicating that the higher the IL-6 values the lower the IGF-I values in the intensive phase (Figure 4).

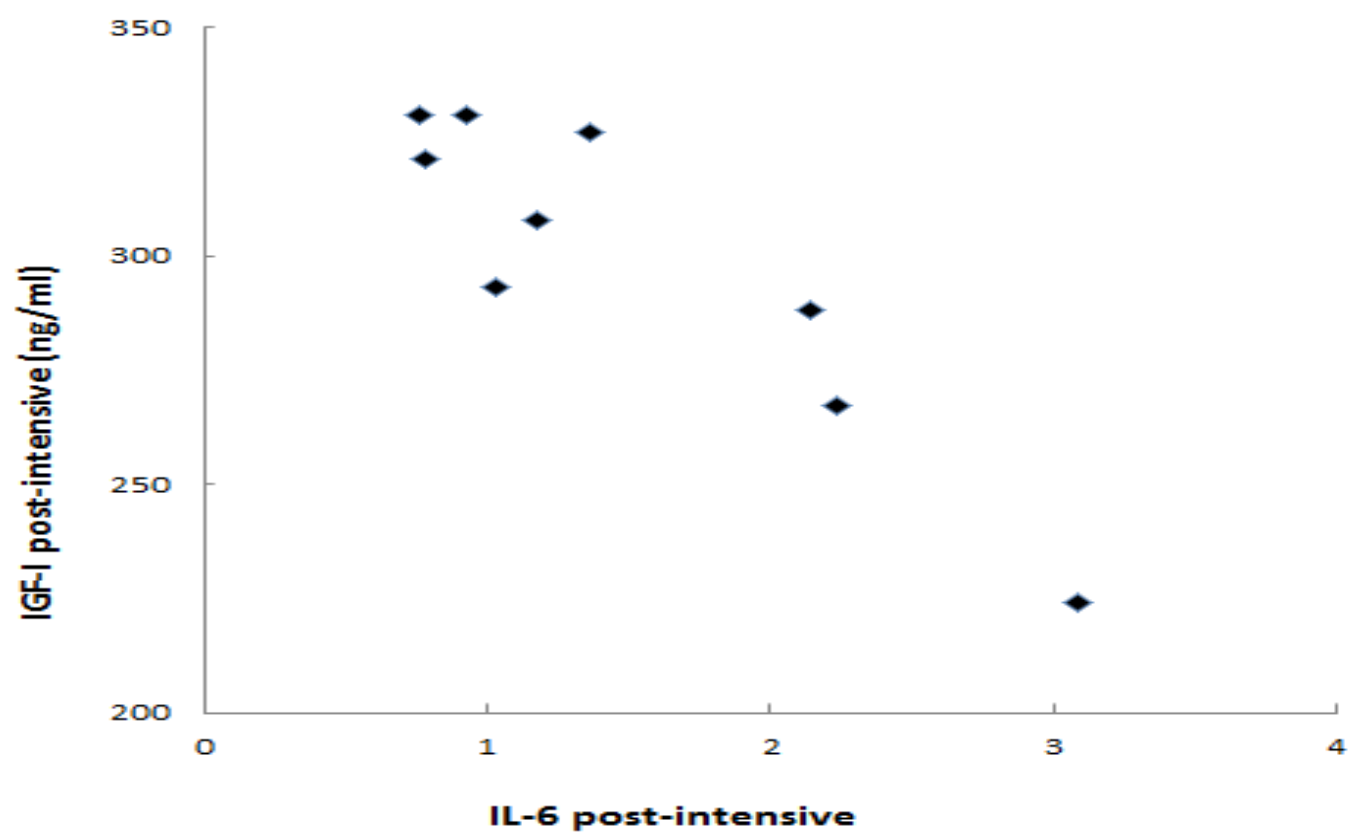

Fig. 4. Correlation between absolute values of post-intensive IGF-I serum concentrations (post-intensive IGF-I) and absolute serum concentrations of post-intensive IL-6 (post-intensive IL-6).

No significant correlations were found between the absolute values of serum IGF-I concentrations and the absolute values of serum cytokine concentrations (IL-6, IL-10, and TNF- $\alpha$ ) in the tapering phase.

Figure 5 shows the variation in the delta IGF-I and delta IGFBP-3 values and their comparison with the IL-10 values presented in this study. It is possible to verify that these three substances increased from the intensive phase to the tapering phase $(\mathrm{p} \leq 0.05)$.

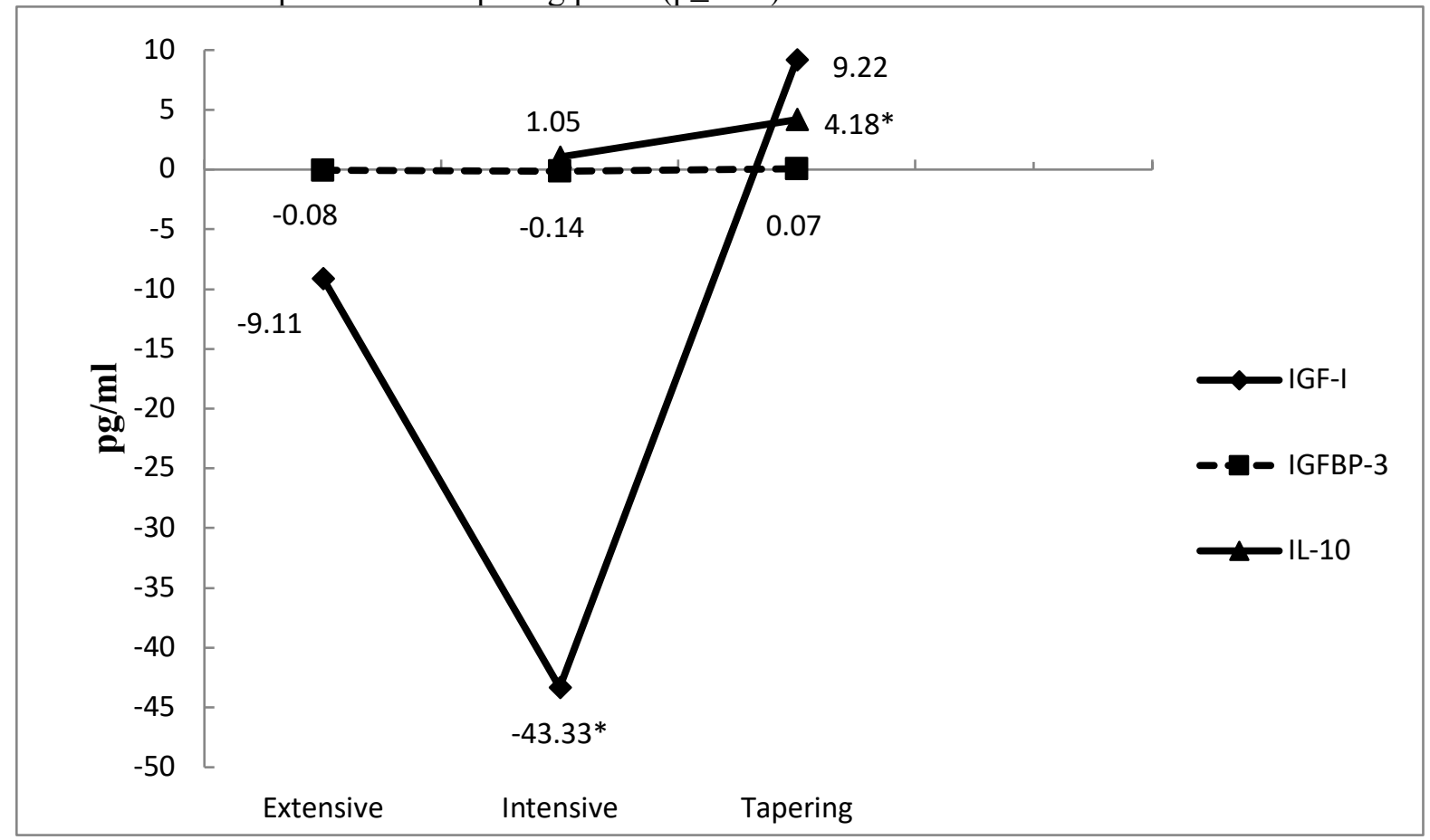

Fig. 5. Kinetics of $\Delta_{\text {IGF-I }}, \Delta_{\text {IGFBP-3, }}$, and $\Delta_{\text {IL-10 }}$ at the different stages of training 


\section{DISCUSSION}

In this study, it was possible to verify a strong and inversely proportional relationship between serum levels of IL-6 and IGF-I during the most intensive phase of training and an increase in serum concentrations of IGF-I and IL-10 when the athletes experienced a reduction in training load (tapering).

When comparing IGF-I values with cytokine values, a catabolic phase was observed during the most intense period of training (intensive phase), characterized by a drop in IGF-I concentrations, and an anabolic phase was verified during tapering, where an increase in IGF-I accompanied by an increase in IL-10 occurred (Figure 5).

It is believed that the clear identification of these two phases (catabolic and anabolic) was partly obtained by controlling the distribution of training loads used throughout the season $(\mathrm{Z1}, \mathrm{Z} 2$, and Z3) and by determining the standardized intensity of the training session for the data collection of the study.

In contrast, Koziris et al. (1999) failed to observe a progressive increase in IGF-I values throughout the season or to identify the two phases - catabolic and anabolic. It is important to emphasize that in the study by Koziris et al. (1999) serum concentrations of cytokines were not obtained, which might have enabled a possible comparison with data from the present study.

The effects of four weeks of training on fitness, self-assessment of physical condition, and circulating IGF-I levels were determined in elite Israeli handball players during preparation for the Junior Handball World Championship (Eliakim, Nemet, Bar-Sela, Higer \& Falk, 2002). Circulating levels of IGF-I declined significantly after the first two weeks of training and returned to baseline levels after four weeks of preparation for the World Championship Eliakim et al. (2002). These results emphasize the idea that a decrease in IGF-I levels may occur, especially in the most intense training period, as verified in the present study.

Regarding the acute effects of training on the serum levels of IGF-I, IGFBP-3, IL-6, IL-10, and TNF- $\alpha$ observed in this study, from the values obtained before and after the standardized training session (pre vs. post), it was possible to identify differentiated kinetics only for the IGF-I values during the intensive phase and for the IL-10 values during the tapering phase.

In the intensive training phase, IGF-I serum concentrations were significantly reduced after the standardized training session, when compared to the values measured at rest before training. In relation to IL-10, an increase in serum concentrations was verified after the training session in the tapering phase, when compared to the values obtained at rest.

Some studies in the literature have demonstrated a decrease in the circulation of the elements that make up the GH/IGF-I axis in adolescents and children in response to intense training sessions (Nemet et al., 2002; Nemet et al., 2003; Scheett et al., 2002; Eliakim, Scheett, Newcomb, Mohan \& Cooper, 2001; Pilz-Burstein, et al., 2010).

In order to test the hypothesis that cytokines may affect the kinetics of the GH/IGF-I axis in response to stimuli from training sessions, Scheett et al. (1999) evaluated the effects of an exercise protocol that consisted of soccer training sessions for prepubescent children that lasted around 1 hour and 30 minutes. The soccer training led to a significant increase in the circulating levels of TNF- $\alpha$ and IL-6. IGF-I levels declined to a lesser extent, but significantly, although IGFBP-1 levels, known to inhibit IGF-I, were substantially increased. For the authors, the increases in circulating levels of cytokines provoked by training sessions can critically alter anabolic agents such as IGF-I and its binding proteins (Scheett et al, 1999).

Petersen \& Pedersen (2005) believe that pre-exercise muscle glycogen content may represent an important stimulus for transcription of the IL- 6 gene, thus the muscle-derived IL- 6 can act as an energetic sensor. It should be said that the biological role of IL-6 from the muscle still needs to be better determined, although the literature presents data supporting the hypothesis that IL- 6 released during muscle contraction acts in a hormone-like manner, with the intention of mobilizing extracellular substrates, which may increase their availability throughout exercise (Petersen \& Pedersen, 2005).

Following this line of reasoning, Febbraio et al. (2003) performed a study to verify the effect of glucose ingestion on the release of IL-6 from the limb required during exercise. From this study, it can be concluded that glucose ingestion during exercise was able to attenuate the release of IL-6, but did not decrease the intramuscular expression of IL-6 mRNA, therefore these data suggest that the release of IL- 6 by muscle contraction during exercise is regulated by the availability of the substrate (Febbraio et al. (2003). 
Data from Petersen \& Pedersen (2005) and from Febbraio et al. (2003) assist in the elaboration of a possible explanation for the negative correlation found between the post-intensive IGF-I and postintensive IL-6 in the present study. The kinetics of IL- 6 during the most intense training period (intensive phase) could determine the decrease in IGF-I, through a direct regulatory action. As well as the fact that GH can directly stimulate the synthesis and secretion of IGF-I, IL-6 could act directly by inhibiting the production of IGF-I. In this sense, IL-6 would act as an energy sensor to mobilize substrates and increase their availability during exercise. For the authors, glycogen deficiency is associated with increases in the local expression of cytokines (IL-6 in muscles), decreased glucose transporters, increased cortisol, and decreased insulin secretion and $\beta$ adrenergic stimulation, and these cytokines specifically act in this process as signals for the hypothalamus that affects the GH/IGF-I axis, consequently reducing IGF-I levels.

In this context, with the objective of measuring the plasma concentrations of IL-6 and IL-15, Kapilevich, Kironenko, Zakharova, Kabachkova \& Orlov, (2017) subjected 160 female rats, divided into a control group $(n=10)$ and experimental group $(n=150)$, to an acute forced swimming test with loads of 5\%,7.5\%, and 10\% of their body weight. According to the authors, IL-6 increases in a delayed manner as it is considered a factor of energy supply. In addition, this cytokine induces fat cleavage and oxidation and participates in the maintenance of glucose homeostasis during exercise (Kapilevich et al., 2017). The kinetics of IL-6 and IL-15 in untrained animals and their delayed effects demonstrate that these cytokines are responsible for the restoration of energy and the overcompensation process. In trained animals the role of these cytokines is to provide the necessary energy to the muscle according to the increase in training loads and increase in intensity (Kapilevich et al., 2017).

In the present study, the swimmers underwent a high level of training, so it is possible that IL-6 acted to provide the necessary energy for the muscles, according to the increase in training intensity in the intensive phase.

On the other hand, IL-10 would act as an anti-inflammatory cytokine, and therefore the elevation of IL-10 observed in this study may have stimulated the increase in IGF-I in the tapering phase, and this factor could play a role in repairing the tissue micro-lesions.

Besides the exercise, others factors can also influence the GH/IGF-I axis, such as a reduction in pre-competition weight, and a high carbohydrates and lipids diet (Galassetti et al., 2006). The dietary recall analysis performed on the adolescent swimmers enables the influence of diet on our results to be excluded as the diet recall was similar at the 3 moments of evaluation, and there were no statistically significant differences in the values of body mass, lean mass, or percentage of fat measured in the swimmers throughout the season

Recent studies have substantially contributed to the endocrinology of exercise. Changes in the anabolic/catabolic balance and inflammatory mediators according to different training sessions in distinct phases of the season may help coaches and athletes in planning training to improve performance (Eliakim \& Nemet, 2013).

Funding: no financing was granted during this study.

Conflicts of interest: the authors state no conflicts of interest regarding this study.

Acknowledgements: sincere thanks are given to the coaching staff and all the athletes of UNAERP Swimming Team, São Paulo, Brazil.

\section{REFERENCES}

Behnke A.R., Wilmore J.H. (1974). Field Methods, Prentice Hall, New Jersey.

Boileau R.A., Lohman T.G. (1985). Slaughter M.H. Exercise and body composition in children and youth. Scandinavian J. Sports Sci.; 7:17-27.

Eliakim A., Cooper D.M., Nemet D. (2014). The GH-IGF-I response to typical field sports practices in adolescent athletes: a summary. Pediatr. Exerc. Sci.; 26 (4): 428-433.

Eliakim A., Nemet D. (2013). Exercise and the GH/IGF-I axis, in: N. Constantini, A.C. Hackney (Eds.), Endocrinology of Physical Activity and Sport. New York: Springer Science + Business Media; pp. 69-83. 
Eliakim A., Nemet D. (2010). Exercise training, physical fitness and the growth hormone-insulin-like growth factor-1 axis and cytokine balance, in: Jürimäe J., Hills A.P., Jürimäe, T. (Eds.). Cytokines, Growth Mediators and Physical Activity in Children during Puberty. Karger Basel:Med Sport Sci.; pp. 128-140.

Eliakim A., Nemet D., Bar-Sela S., Higer Y, Falk B. (2002). Changes in circulation IGF-I and their correlation with self-assessment and fitness among elite athletes. Int. J. Sports Med.;23 (8): 600603.

Eliakim A., Scheett T.P., Newcomb R., Mohan S., Cooper D.M. (2001). Fitness, training, and the growth hormone - insulin-like growth factor I axis in prepubertal girls, J. Clin. Endocrinol. Metab.; 86 (6): 2797-2802.

Esteve-Lanao J., Juan A.F.S., Earnest C.P., Foster C., Lucia A. (2005). How do endurance runners actually train? Relationship with competition performance, Med. Sci. Sports Exerc.; 37 (3): 496504.

Febbraio M.A., Steensberg A., Keller C., Starkie R.L., Nielsen H.B., Krustrup P., Ott P., Secher N.H., Pedersen B.K. (2003). Glucose ingestion attenuates interleukin-6 release from contracting skeletal muscle in humans. J. Physiol.;549: 607-612.

Galassetti P., Larson J., Iwanga K., Salsberg S., Eliakim A., Pontello A. (2006). Effect of a high fat meal on the growth hormone response to exercise in children, J. Pediatr. Endocrinol. Metab; 19: 777786.

Kapilevich L.V., Kironenko T.A., Zakharova A.N., Kabachkova A.V., Orlov S.N. (2017). Level of interleukins IL-6 and IL-15 in blood plasma of mice after forced swimming test. Bulletin of Experimental Biology and Medicine; 163 (1): 14-17.

Koziris L.P, Hickson R.C., Chatterton Jr R.T., Groseth R.T., Christie J.M, Goldflies D.G., Unterman T.G. (1999). Serum levels of total and free IGF-I and IGFBP-3 are increased and maintained in long-term training, J. Appl. Physiol.; 86 (4): 1436-1442.

Maglischo E.W. (2010). Swimming even faster. Barueri: Manole.

Marchioni D.M.L, Slater B., Fisberg R.M. (2004). Application of the dietary reference intakes in the evaluation of nutrient intake for individuals. Rev. Nutr.; 17 (2): 207-216.

Martinelli Jr. C.E., Custódio R.J, Oliveira M.H.A. (2008). Physiology of the GH axis-IGF system. Arq. Bras. Endocrinol. Metab.; 52 (5): 717-725.

Moldoveanu A.I, Shephard R.J., Shek P.N. (2001). The cytokine response to physical activity and training. Sports Med.; 31: 115-44.

Nemet D., Eliakim A. (2010). Growth hormone-insulin-like growth factor-1 and inflammatory response to a single exercise bout in children and adolescents, in: Jürimäe J., Hills A.P, Jürimäe, T. (Eds.). Cytokines, Growth Mediators and Physical Activity in Children during Puberty. Karger, Basel: Med Sport Sci.; pp. 141-155.

Nemet D., Oh Y., Kim H.S., Hill M.A., Cooper D.M. (2002). Effect of intense exercise on inflammatory cytokines and growth mediators in adolescent boys. Pediatrics. (4): 681-689.

Nemet D., Rose-Gottron C.M., Mills P.J., Cooper D.M. (2003). Effect of water polo practice on cytokines, growth mediators, and leukocytes in girls. Med. Sci. Sports Exerc.; 35 (2): 356-363.

Nindl B.C., Kraemer W.J., Marvs J.O., Arciero P.J., Dohi K., Kellogg M.D., Loomis G.A. (2001). Overnight responses of the circulating IGF-I system after acute heavy-resistance exercise. J. Appl. Physiol.; 90 (4): 1319-1326.

Petersen A.M., Pedersen B.K. (2005). The anti-inflammatory effect of exercise. J. Appl. Physiol.; 98 (4): 1154-1162.

Pilz-Burstein R., Ashkenazi Y., Yaakobovitz Y., Cohen Y., Zigel L., Nemet D. Shamash N., Eliakim A. (2010). Hormonal response to taekwondo fighting simulation in elite adolescent athletes. Eur. J. Appl. Physiol.; 1(10): 1283-1290.

Scheett T.P, Mills P.J., Ziegler M.G., Stoppani J., Cooper D.M. (1999). Effect of Exercise on Cytokines and Growth Mediators in Prepubertal Children. Pediatr. Res.; 46 (4): 429-440.

Scheett T.P, Nemet D., Stoppani J., Maresh C.M., Newcomb R., Cooper D.M. (2002). The effect of endurance-type exercise training on growth mediators and inflammatory cytokines in pre-pubertal and early pubertal males. Pediatr. Res.; 52 (4): 491-497.

Seiler K.S., Kjerland G.O. (2006). Quantifying training intensity distribution in elite endurance athletes: is there evidence for an "optimal" distribution? Scand. J. Med. Sci. Sports.; 16: 49-56. 
Silva C.C., Goldberg T.B.L., Teixeira A.S., Marques I. (2004). Does physical exercise potentiate or compromise the longitudinal growth of children and adolescents? Myth or truth? Brazilian Journal of Sports Medicine.; 10 (6): 520-524.

Tanner J.M. (1962). Growth at Adolescent. Oxford, England: Blackwell Scientific Publications.

Tourinho Filho H., Pires M., Puggina E.F., Papoti M., Barbieri R., Martinelli Jr C.E. (2017). Serum IGF-I, IGFBP-3 and ALS concentrations and physical performance in young swimmers during a training season. Growth Hormone \& IGF Research.; 32: 49-54.

\section{CITATION OF THIS ARTICLE}

Pires, M., Hugo Tourinho Filho, H.T., Kohama, E., Fornel, R., Custódio, R., Carlos Eduardo Martinelli Junior, C.E.M. Kinetics of cytokines IL-6, IL-10 and TNF- $\alpha$ and their relationship with serum IGF-I and IGFBP-3 concentrations in adolescent swimmers throughout a training season. International Journal of Sport, Exercise \& Training Sciences-IJSETS, 6(4), 138-148. DOI:10.18826/useeabd.843434 